\title{
alfabetizAR: Uma Aplicação Móvel com base na Realidade Aumentada como Ferramenta de Apoio no Processo de Alfabetização de Portadores de Síndrome de Down
}

\author{
Dayane R. de Souza ${ }^{1}$, Genarde M. Trindade ${ }^{2}$, Bruno A. Bonifácio ${ }^{1}$, Priscila S. \\ Fernandes ${ }^{1}$ \\ ${ }^{1}$ Instituto de Ciências Exatas e Tecnologia - Universidade Federal do Amazonas \\ (UFAM) \\ Rua Nossa Senhora do Rosário - 69103-128 - Itacoatiara - AM - Brazil \\ ${ }^{2}$ Centro de Estudos Superiores de Itacoatiara- Universidade do Estado do Amazonas \\ (UEA) \\ \{dayanerosas, genardemacedo, brunnoboni, pry.bila \}egmail.com
}

\begin{abstract}
This paper presents the assessment and validation of an application using augmented reality technology for the teaching of letters and words for individuals with Down Syndrome. The main goal is to support the literacy process of people with Down Syndrome. To conduct this research, we use an empirical based methodology. The results show that the application is useful to aid in the process of literacy for people with Down Syndrome.
\end{abstract}

Resumo. Este trabalho apresenta o desenvolvimento e validação de um aplicativo usando a tecnologia de Realidade Aumentada para o ensino de letras e palavras para portadores de Síndrome de Down. O objetivo principal é apoiar o processo de alfabetização de pessoas com Síndrome de Down. A metodologia utilizada neste trabalho é baseada em estudos experimentais. Os resultados da pesquisa mostram que o aplicativo é útil para auxiliar no processo de alfabetização de pessoas portadoras de Síndrome de Down.

\section{Introdução}

A Tecnologia da Informação (TI) vem sendo utilizada pela sociedade como ferramenta facilitadora nas tarefas diárias, inclusive na área educacional [Cardoso et al., 2014]. Com o crescimento da TI surgiu a Realidade Aumentada (RA). De acordo com Kirner (2008), a RA mistura objetos virtuais ao mundo real, adotando técnicas de visão computacional. Dentre os diversos recursos tecnológicos educacionais, pode-se apontar a RA como uma das tecnologias que pode auxiliar na construção do conhecimento, possibilitando a interatividade do usuário [Fernandes et al., 2014]. Araújo (2009) afirma que a partir da projeção de imagens, chamados marcadores, a tecnologia de RA possibilita uma maior interação entre o aluno e o conteúdo apresentado pelo professor, facilitando a compreensão dos assuntos abordados em sala de aula.

A Síndrome de Down (SD) se caracteriza por uma alteração genética. Embora o portador de SD apresente algumas dificuldades, ele pode realizar atividades diárias da mesma forma que outra pessoa que não possui a síndrome [Schawartzman, 1999]. No 
processo de ensino-aprendizagem são indispensáveis condições especiais para estimular adequadamente o aprendizado do portador de $\mathrm{SD}$, visto que o processo de aprendizagem não acontece com facilidade [Bassani, 2012]. A utilização de recursos tecnológicos para poradores de SD torna-se uma ferramenta importante no sentido de simplificar e socializar a produção dos conhecimentos culturalmente construídos e que se encontravam fora do alcance desse público [Carsten, 2013].

Neste contexto, o presente trabalho apresenta um aplicativo com base na RA, chamado alfabetizAR, que visa auxiliar no processo de alfabetização de portadores de Síndrome de Down. Neste trabalho são descritos o desenvolvimento e avaliação do aplicativo, seguindo a metodologia de pesquisa adotada.

A metodologia utilizada nessa pesquisa, parcialmente adaptada de Mafra et al., (2006), é baseada em estudos experimentais, e compreende cinco etapas: (1) Revisão da Literatura: foram buscados subsídios teóricos sobre as abordagens do desenvolvimento cognitivo de acordo com as teorias de Vigotsky e trabalhos relacionados a pesquisa; (2) Proposta Inicial: a técnica utilizada para levantamento de requisitos foi a entrevista semi-estruturada com stakeholders, sendo a diretora e a pedagoga da instituição onde foi aplicado o estudo, os entrevistados puderam discorrer sobre suas experiências com alunos portadores de Síndrome de Down considerando as necessidades especificas de ensino desses alunos o que permitiu gerar a proposta inicial do aplicativo. (3) Desenvolvimento do Aplicativo: nessa etapa foram executadas atividades para o desenvolvimento do aplicativo, como projeto e implementação; (4) Validação do aplicativo com profissionais da área educacional: Nessa etapa o aplicativo foi apreciado por professores e pedagogos visando identificar a adequação do alfabetizAR para portadores de Síndrome de Down; (5) Estudo de Observação: para aprofundar a compreensão de como os usuários utilizam o aplicativo, foi realizado um estudo de observação. A partir da observação foi possível identificar possíveis problemas de interação, possibilitando o aprimoramento do alfabetizAR.

\section{Fundamentação Teórica}

Existem diversos trabalhos na literatura que propõem a utilização de recursos tecnológicos para apoiar o processo de ensino-aprendizagem. Dentre estes recursos estão jogos educacionais, aplicativos móveis, ambientes virtuais, e a realidade aumentada [Soffa e Torres, 2009]. A RA é uma tecnologia que permite que objetos reais e virtuais coexistam em um mesmo espaço físico [Kirner 2004]. Esse fator enriquece o cenário e proporciona um nível maior de interação do usuário com a aplicação. A vantagem de utilizar a RA na educação, é que algumas técnicas exigem poucos recursos, por exemplo, dispositivos móveis que possuam câmera [Tori, 2009].

A RA no contexto educacional possibilita uma nova experiência ao aluno e o faz interagir com o ambiente virtual sem a necessidade de possuir grandes conhecimentos de informática, aumentando sua visão do mundo real, uma vez que essa ferramenta tecnológica possibilita a inserção de objetos virtuais no ambiente real [Prezotto et al., 2013]. É possível encontrar diferentes iniciativas na literatura sobre a RA aplicando abordagem no contexto educacionail considerando alunos com diferentes perfis, incluindo os portadores de SD. Ainda assim, para agregar valor aos resultados, é 
VI Congresso Brasileiro de Informática na Educação (CBIE 2017)

Anais do XXVIII Simpósio Brasileiro de Informática na Educação (SBIE 2017)

importante analisar quais os impactos quanto ao uso de uma determinada tecnologia na educação especial [Paula, et al., 2011].

\subsection{Portadores de Sídrome de Down}

Indivíduos com Síndrome de Down manifestam características físicas e comportamentos em comum. Schwartzman (1999) afirma que ocorre um atraso em todas as áreas do desenvolvimento do portador de SD, entretanto, não existe um padrão pré-definido de desenvolvimento do portador dessa síndrome, pois o desenvolvimento cognitivo não depende necessariamente da alteração cromossômica, mas da influência que a sociedade exerce. Segundo Romero (2014), o desenvolvimento da criança com SD em geral é semelhante ao de crianças sem a síndrome, as etapas de aprendizado são atingidas, embora em um ritmo mais lento. Além disso, o portador de SD desenvolve diferentes maneiras para manifestar sua capacidade de abstração e simbolização [Oliveira, 2007].

Para Pacheco e Oliveira (2011), é importante que desde a infância o portador de SD seja estimulado pedagogicamente, com atividades que enfatizem aspectos cognitivos, como percepção, atenção, memória e linguagem; no intuito de trabalhar as limitaçõe. Esses estímulos pedagógicos ajudam o portador de SD a ter um contato mais direto com o meio em que vive.

\subsection{Trabalhos Relacionados}

A seguir serão apresentados três trabalhos correlatos, os quais estão relacionados a aplicativos educaconais no contexto da tecnologia de Realidade Aumentada na educação de portadores de Síndrome de Down (SD), que serviram como suporte para realização deste trabalho.

Dos Santos et al., (2017) apresentam uma aplicação móvel, no projeto foi desenvolvido um protótipo do jogo físico Numicon utilizado na educação de portadores de SD para auxiliar no processo de aprendizagem do conteúdo de matemática básica. $\mathrm{O}$ Numi mobile utiliza os recursos dos dispositivos móveis na plataforma Android para auxiliar crianças, na faixa etária de um a treze anos de idade. O resultado da validação forneceu dados que mostram a relevância e o potencial do Numi.

Rodrigues e Félix (2014) propõem um software chamado ABCÊ Bulir, o software auxilia no processo de alfabetização e desenvolvimento cognitivo de portadoes de Síndrome de Down. O trabalho apresentado trata-se de uma aplicação para desktop que contém atividades de software educacional, onde o usuário pode visualizar as atividades de forma dinâmica, podendo realizar as atividades proposta na aplicação. $\mathrm{O}$ software é composto por três módulos, dos quais dois são para execução de atividades de conteúdo educacional.

Veneziano et al., (2013) apresentam o software para desktop chamado Programa Participar, trata-se de um software educacional de apoio à alfabetização de jovens e adultos com deficiência intelectual. $\mathrm{O}$ software possui recursos multimídia de áudio e vídeo, facilitando a interação do professor com o aluno especial. O processo de validação do software foi realizado com estudantes portadores de SD. 
A Tabela 1 apresenta o comparativo entre o aplicativo alfabetizAR proposto neste trabalho com as aplicações dos trabalhos correlatados para portadores de Síndrome de Down. A análise comparativa das aplicações foi realizada utilizando a técnica feature analysis. Esta técnica objetiva comparar tecnologias específicas de forma qualitativa, utilizando critérios específicos [Travassos et al., 2002]. Foram considerados os seguintes critérios: (1) natureza da aplicação a ser avaliada; (2) utiliza áudio; (3) é voltada para a alfabetização de portadores de SD; (4) é mobile, o critério mobile foi escolhido afim de que os alunos portadores de SD utilizem o alfabetizAR tanto na escola, quanto em casa para ajudá-los nas taefas escolares; (5) utiliza a tecnologia de RA; (6) foi avaliada com o público alvo. O "X" significa que a aplicação atende os critérios estabelecidos. Dessa forma, é possível perceber que o alfabetizAR (aplicativo proposto nesta pesquisa) atendeu todos os critérios.

Tabela 1. Feature Analysis das aplicações educacionais

\begin{tabular}{|l|c|c|c|c|c|}
\hline \multicolumn{1}{|c|}{ Aplicações } & Áudio & $\begin{array}{c}\text { Alfabetização de } \\
\text { Portadores de SD }\end{array}$ & Mobile & RA & Avaliada \\
\hline Numi & & $\mathrm{X}$ & $\mathrm{X}$ & & $\mathrm{X}$ \\
\hline ABCÊ Bulir & $\mathrm{X}$ & $\mathrm{X}$ & & & $\mathrm{X}$ \\
\hline Programa Participar & $\mathrm{X}$ & $\mathrm{X}$ & & & $\mathrm{X}$ \\
\hline alfabetizAR & $\mathrm{X}$ & $\mathrm{X}$ & $\mathrm{X}$ & $\mathrm{X}$ & $\mathrm{X}$ \\
\hline
\end{tabular}

\section{O Aplicativo alfabetizAR}

O aplicativo alfabetizAR tem como objetivo apoiar alunos portadores de SD no processo de alfabetização, possibilitando que os usuários vejam objetos bidimensionais (2D) relacionados às letras do alfabeto. Além disso, o aplicativo permite ouvir o áudio correspondente à letra e à palavra do objeto apresentado, proporcionando uma melhor assimilação e aprendizado do conteúdo. Nesse contexto, essa ferramenta tecnológica poderá ser um recurso pedagógico que auxiliará professores e alunos portadores de Síndrome de Down não alfabetizados. Alunos portadores de SD, professores podem acessar o aplicativo alfabetizAR por meio de um smartphone ou tablet que utilize a plataforma Android. Sem a necessidade de realização de login, a ferramenta poderá ser utilizada para auxiliar nas atividades pedagógicas.

A modelagem do aplicativo foi realizada com base na entrevista realizada com os skateholders na Associação de Pais e Amigos dos Excepcionais (APAE) de Itacoatiara, no estado do Amazonas. Para o desenvolvimento do aplicativo alfabetizAR foi feito um protótipo evolucionário, que consistiu em produzir um modelo inicial e refiná-lo ao longo das várias fases de desenvolvimento até atingir a forma final, foram utilizadas as ferramentas Unity 3D, Vuforia, Android SDK (Software Development Kit) e Android NDK (Native Development Kit). A linguagem C\# e JavaScript foram utilizadas para implementação do aplicativo no Unity. Essas ferramentas tecnológicas foram escolhidas visando atender os requisitos do aplicativo proposto neste trabalho.A tela inicial do aplicativo exibe algumas funções, onde o usuário poderá escolher qual opção deseja, tais como: (1) Iniciar, onde o usuário será direcionado a câmera de Realidade Aumentada e poderá colocar o marcador diante da câmera do smartphone ou tablet, e poderá ver os objetos bidimensionais sobrepostos no ambiente real, esse botão chama mais atenção em forma de movimento para o aluno; (2) botão Menu, o usuário será direcionado a telas que contém os botões Sobre, contendo informações do aplicativo; (3) botão Ajuda, contém as instruções de uso do aplicativo através de áudio 
VI Congresso Brasileiro de Informática na Educação (CBIE 2017)

Anais do XXVIII Simpósio Brasileiro de Informática na Educação (SBIE 2017)

para o alunos não alfabetizados e no formato de texto para os professores; (4) botão Autores, contém informações dos autores do aplicativo; (5) botão Marcador, onde o usuário poderá fazer o download dos marcadores através do Google Drive; (6) botão Sair, o usuário poderá sair do aplicativo. Os alunos portadores de SD não alfabetizados poderão usar o aplicativo com o apoio do professor ou responsável. A Figura 4 mostra as principais telas do aplicativo.

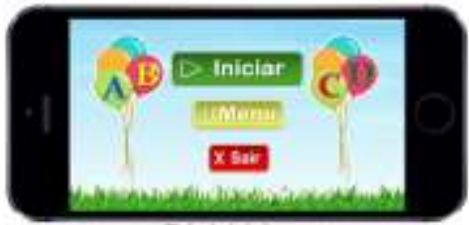

Tela iaicial

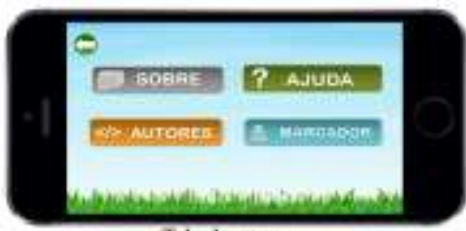

Tela de menu

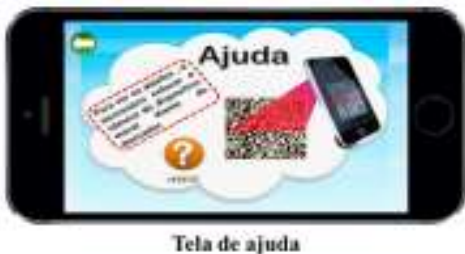

Tela de ajudu

Figure 4. Telas principais do aplicativo alfabetizAR

A Figura 5 mostra algumas letras, palavras e objetos 2D sobreposto ao usuário quando a câmera do dispositivo móvel reconhece o marcador previamente cadastrado no banco de dados.
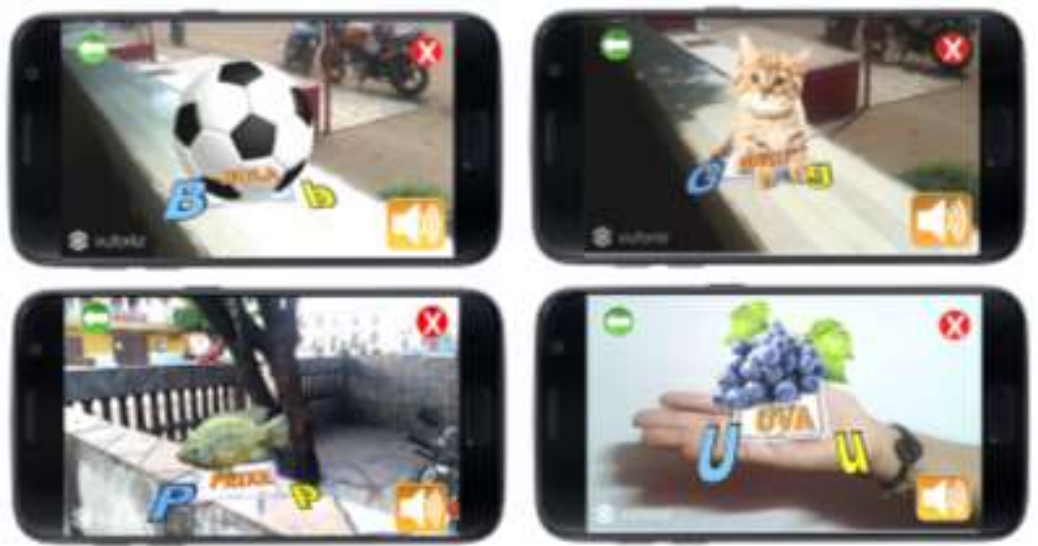

Figure 5. Objetos 2D sobrepostos ao usuário

\section{Avaliação do Aplicativo alfabetizAR}

Esta seção apresenta os dois estudos realizados visando avaliar o aplicativo: a validação do aplicativo foi realizada com seis participantes, sendo cinco professores e uma pedagoga que atuam diretamente com alunos portadores de $\mathrm{SD}$, funcionários da APAE Itacoatiara; E o estudo de observação realizado com alunos entre oito a quarenta e seis anos de idade não alfabetizados, todos com diagnóstico de Síndrome de Down.

\subsection{Validação do alfabetizAR}

A Etapa 4 da metodologia consistiu em um estudo de validação do aplicativo. O objetivo deste estudo foi avaliar a percepção de profissionais da área educacional visando identificar a adequação do alfabetizAR para portadores de Síndrome de Down. A pergunta a ser respondida foi "O aplicativo está adequado para o público alvo?". O planejamento do estudo foi feito visando validar o alfabetizAR de forma qualitativa, por meio da percepção dos professores e da pedagoga. 
No planejamento foram elaborados os seguintes documentos: (1) Termo de Consentimento Livre e Esclarecido (TCLE). (2) Formulário de Caracterização do Participante, que continha questões que deveriam ser respondidas pelos professores em relação ao seu conhecimento e experiência em aplicativos educacionais. E (3) Questionário Pós-Teste baseado no Technology Acceptance Model (TAM) - Modelo de Aceitação Tecnológica [Davis et al., 1989]. A proposta básica do TAM é prover uma base para traçar o impacto de variáveis em crenças internas, atitudes e intenções, para determinar: Facilidade Percebida de Uso e Utilidade Percebida como uso de aplicativos educacionais. Esse modelo é aplicável ao problema desta pesquisa por ser específico para os usuários que utilizam aplicativos ou softwares educacionais e possui vantagem forte nas bases teóricas, além do apoio empírico através de validações da tecnologia proposta. No questionário TAM, os participantes informaram o seu grau de concordância em relação às afirmações sobre a utilidade e sobre facilidade de uso do aplicativo alfabetizAR. Além disso, o questionário continha perguntas específicas sobre as características do aplicativo quanto à adequação para o púbico alvo, se o alfabetizAR poderia ser utilizado por pessoas que não são Portadores de Necessidades Educativas Especiais, e se o aplicativo poderia ser utilizado como ferramenta pedagógica para auxiliar na alfabetização.

\subsubsection{Execução do Estudo}

Foi alocada uma sala da APAE Itacoatiara para a realização do estudo. Primeiramente foram explicados os procedimentos da pesquisa, caso o participante concordasse em participar, recebia e assinava o TCLE. Após isso, foi entregue o Formulário de Caracterização do Participante e solicitado que os participantes o preenchessem. Foram disponibilizados 4 tablets, 2 smartphones, e 26 marcadores para os participantes realizarem as atividades. O estudo foi realizado em 20 minutos, correspondente ao horário de intervalo das aulas. Todos os participantes puderam interagir individualmente com o aplicativo. Os pesquisadores registraram as sugestões de melhorias e percepções citadas pelos participantes.

\subsubsection{Análise dos Resultados}

A caracterização dos participantes foi auto-avaliativa. Os dados coletados pelo documento de caracterização com os professores e a pedagoga mostraram que dois participantes possuíam baixa experiência no uso de aplicativos educativos e quatro participantes possuíam alta experiência. Serão descritos a seguir dados qualitativos coletados:

(1) O alfabetizAR pode ser utilizado como ferramenta pedagógica para auxiliar o processo de ensino-aprendizagem na Alfabetização? Todos os participantes concordam totalmente conforme relato do Participante 6: "Não há dúvida que esse aplicativo pode ser uma ferramenta pedagógica", e o Participante 3: "Esse aplicativo pode com certeza ser usado para auxiliar a alfabetização dos alunos, seria muito bom que os pais também soubessem desse aplicativo, assim eles poderiam ajudar seus filhos nas atividades escolares de seus filhos também".

(2) O alfabetizAR está adequado para alunos Portadores de Síndrome de Down? Todos participantes concordam totalmente que o aplicativo está adequado para alunos 
portadores de Síndrome de Down, conforme relato do Participante 6: "Com os áudios e com as imagens reais acredito que os alunos poderão sim aprender o alfabeto, pois o aplicativo poderá prender a atenção deles, além disso, atualmente os alunos gostam muito de tecnologia, mas seria interessante incluir a repetição de áudio no aplicativo, pra ficar mais fácil associar as letras com os objetos".

Apesar das sugestões de melhoria, os dados mostram indícios de que o aplicativo alfabetizAR é adequado para o público alvo, pois a maioria dos participantes concorda totalmente sobre a facilidade e utilidade de uso do aplicativo. Nesse sentido o aplicativo pode ser uma ferramenta pedagógica que auxiliará professores e alunos portadores de SD dentro e fora da sala de aula.

\subsection{Melhorias Realizadas a partir dos Resultados}

Os resultados obtidos no estudo de validação com professores e a pedagoga permitiram revisar e acrescentar pequenas melhorias e novas funcionalidades para a segunda versão do aplicativo, como repetição do áudio, download dos marcadores, o tamanho das letras e do objeto sobreposto.

Os dados qualitativos sobre a percepção dos profissionais sobre a facilidade de uso e utilidade foram primordiais para a validação do aplicativo, dessa forma o aplicativo está adequado para o púbico alvo. Os dados qualitativos formaram uma importante base para o aprimoramento do aplicativo alfabetizAR.

\subsection{Estudo de Observação com Alunos Portadores de Síndrome de Down}

A Etapa 5 da metodologia consistiu em um estudo de observação. O objetivo deste estudo foi observar se o aplicativo foi utilizado de maneira esperada pelos alunos portadores de Síndrome de Down (SD). A pergunta a ser respondida foi "O aplicativo foi utilizado de maneira esperada?", a fim de observar as interações e dificuldades encontradas pelos alunos com o uso do aplicativo. Foram selecionados quinze alunos não alfabetizados: 3 alunos do $1^{\circ}$ Ano Matutino (8 à 10 anos); 5 alunos do Projeto Talento "A" Vespertino (17 à 20 anos); 7 alunos do Projeto Dança, Coral e Arte Vespertino (18 à 46 anos). O motivo de se aplicar o estudo com os alunos portadores de SD de idades distintas é que o portador de Síndrome de Down possui idade cronológica diferente da idade funcional, desse modo, não se deve esperar uma resposta equivalente à resposta de um individuo que não possui a síndrome [Silva, 2002].

\subsubsection{Execução do Estudo}

No estudo de observação foram selecionadas algumas salas de aula do horário matutino e vespertino, em todas as salas há alunos Portadores de Necessidades Educativas Especiais. Entretanto, o estudo de observação foi realizado somente com os alunos portadores de Síndrome de Down não alfabetizados, cursando as seguintes séries: $1^{\circ}$ ano, Projeto Talento "A", e Projeto Artes "B".

Foi disponibilizado um tablet por aluno para realização do teste. Cada aluno ficou no máximo cinco minutos utilizando o aplicativo. $\mathrm{O}$ estudo foi realizado com um aluno por vez. A interação dos participantes com o aplicativo, além de gestos e ações foram registrados como critérios para a validação do aplicativo no estudo de 
VI Congresso Brasileiro de Informática na Educação (CBIE 2017)

Anais do XXVIII Simpósio Brasileiro de Informática na Educação (SBIE 2017)

observação. Os pesquisadores também registraram as dificuldades vivenciadas pelos participantes.

\subsubsection{Análise dos Resultados}

Dois alunos entre 8 a 10 anos de idade interagiram muito bem com o aplicativo e um aluno precisou de ajuda para ajustar a câmera do dispositivo móvel em direção ao marcador. O Aluno 1 e Aluno 2 interagiram muito bem com aplicativo, conseguiram trocar os marcadores sem ajuda dos pesquisadores e professores para ver as letras, as sílabas e os objetos, além disso eles reconheceram os objetos sobrepostos. O Aluno 1 percebeu que todas as vezes que seu dedo ficava na frente da câmera o objeto não era sobreposto, desta forma, ele evitava que dedo ficasse na frente da câmera. O Aluno 3 precisou de ajuda para ajustar câmera do dispositivo móvel em direção ao marcador, a cada surgimento dos objetos ele observava atentamente.

Três alunos entre 17 a 20 anos de idade interagiram muito bem com o aplicativo e dois alunos precisaram de ajuda. O Aluno 4 e o Aluno 5 tiveram um boa interação com o alfabetizAR, eles manusearam o aplicativo sem dificuldades, olhavam atentamente as letras, as sílabas e os objetos que surgiam. O Aluno 4 sorria toda vez que surgia um novo objeto, e demonstrava estar estar gostando bastante do aplicativo. Já o Aluno 5 repetia o nome dos objetos e as letras quando surgiam e eram ouvidas pelo áudio. O Aluno 7 também precisou de ajuda durante a atividade, pois estava colocando o dedo repetidamente na câmera do tablet, o que dificultou o reconhecimento do marcador e consequentemente o não surgimento do objeto. O Aluno 8 interagiu bem com o aplicativo, repetiu o nome dos objetos e das letras que surgiam na tela e no áudio, mas logo quis parar de usar o aplicativo.

Seis alunos entre 18 a 46 anos de idade tiveram uma boa interação com o aplicativo e um aluno precisou inicialmente de ajuda para ajustar câmera do dispositivo móvel em direção ao marcador. A maioria dos alunos falava o nome das letras e dos objetos, repetindo o que tinham ouvido. Eles reconheceram e associaram bem as imagens que eram sobrepostas, trocavam os marcadores sem dificuldades, observavam atentamente, tocavam no botão de repetição de áudio. A Figura 7 mostra as imagens da interação dos alunos com oapliativo alfabetizAR coletadas a partir do estudo de observação.
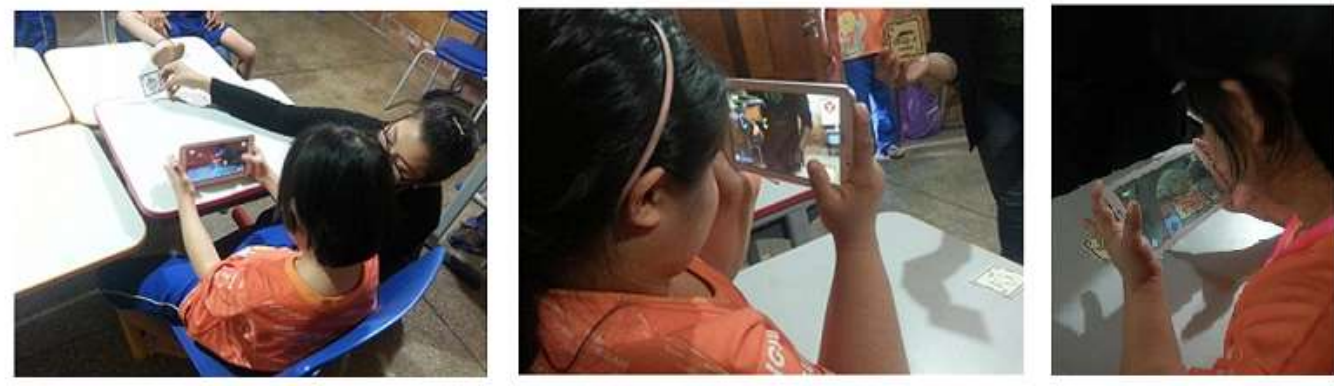

Figure 7. Alunos interagindo com o aplicativo alfabetizAR

\subsubsection{Conclusões e Lições Aprendidas do Estudo de Observação}

Por meio do estudo de observação foi possível obter um conjunto de informações detalhadas em relação à utilização do alfabetizAR pelos alunos portadores de Síndrome 
de Down. Isso permitiu identificar pontos fortes do aplicativo. Dessa forma, dos quinze alunos que fizeram o teste, somente três precisaram de ajuda, e somente um aluno abandonou o aplicativo. Nesse sentido, através do estudo foi possível observar que o aplicativo foi utilizado de maneira esperada pelos alunos portadores de Síndrome de Down. No entanto, devido à pequena amostra, a homogeneidade e o tempo limitado para o teste com os alunos, é recomendado repetir o estudo no futuro com mais alunos e com uma amostra mais heterogênea.

\section{Conclusões e Perspectivas Futuras}

Neste artigo foram apresentatos o desenvolvimento e a validação do aplicativo alfabetizAR. A validação foi realizada com professores e pedagogos e o estudo de observação com alunos portadores de Síndrome de Down. Os resultados da pesquisa mostram indícios que o aplicativo é adequado para o público alvo e que é útil para apoiar no processo de alfabetização de pessoas portadoras de Síndrome de Down.

O alfabetizAR é um aplicativo com base na Realidade Aumentada para apoiar o processo de alfabetização de portadores de Síndrome de Down. O aplicativo possibilita que os usuários vejam objetos bidimensionais relacionados às letras do alfabeto. Nesse contexto, essa ferramenta tecnológica é um recurso pedagógico que poderá auxiliar professores e alunos portadores de Síndrome de Down. A principal razão de se utilizar a RA para portadores de SD é poder estimular e facilitar a aquisição do conhecimento por parte do aluno, além de ajudar o docente em suas práticas pedagógicas

Como sugestão de trabalhos futuros estão: (1) Inserção de novas funcionalidades, tais como atividades complementares para verificar se o aluno está de fato assimilando os conteúdos propostos no aplicativo, adicionar objetos relacionados a numerais e cores; (2) Melhorar a interatividade dos objetos sobrepostos, utilizando recursos de vídeos e objetos em Graphics Interchange Format (GIFs); (3) Realizar um estudo com uma amostra maior e mais heterogênea; (4) Realizar um estudo para avaliar o impacto do aplicativo alfabetizAR no processo de ensino-aprendizagem.

\section{Referências}

Araújo, D. (2009). "Uso de Realidade Aumentada como Ferramenta Complementar ao Ensino das Principais Ligações Entre Átomos", VI Workshop de Realidade Virtual e Aumentada, Universidade de Santa Cecília.

Bassani, C. (2012). "A Síndrome de Down e as Dificuldades de Aprendizagem”, Anhanguera Educacional Unidade Taboão da Serra, São Paulo.

Cardoso, R., et al. (2014). "Uso da Realidade Aumentada em Auxílio à Educação", Computer on the Beach, p. 330-339.

Carsten, G. (2013). "A Utilização do Computador na Alfabetização do Aluno com Deficiência Intelectual”.

Davis, F. et al. (1989). "User acceptance of computer technology: a comparison of two theoretical models". Management science, v.35, n. 8, p. 982. 
VI Congresso Brasileiro de Informática na Educação (CBIE 2017)

Anais do XXVIII Simpósio Brasileiro de Informática na Educação (SBIE 2017)

dos Santos, Pedro C., et al. (2017). "Aplicativo Android Numi: Um Auxílio para o Ensino de Matemática para as Crianças com Síndrome de Down", Anais do Computer on the Beach, 317-326.

Kirner, C. (2004). "Mãos colaborativas em ambientes de Realidade Misturada", $1^{\circ}$ Workshop de Realidade Aumentada, Piracicaba, SP (pp. 1-4).

Kirner, C., Kirner, T. G. (2008). "Virtual reality and augmented reality applied to simulation visualization", Simulation and Modeling: Current Technologies and Applications. IGI Global.

Mafra, S. et al. (2006). "Aplicando uma metodologia baseada em evidência na definição de novas tecnologias de software”, 20o Brazilian Simpósio Brasileiro de Engenharia de Software (SBES).

Oliveira, Ema P.(2007). "Alunos sobredotados: A aceleração escolar como resposta educativa". Universidade do Minho.

Pacheco, W. S.; Oliveira, M. S. (2011). "Aprendizagem e desenvolvimento da criança com síndrome de Down: representações sociais de mães e professoras". Ciência e Cognição.

Paula, M., et al. (2011). "Utilização de jogos para pessoas com necessidades educativas especiais: uma analise experimental”, XXII SBIE - XVII WIE - Aracajú - SE.

Prezotto, E. et al. (2013). "Realidade aumentada aplicada a educação", encontro Anual de Tecnologia da Informação, 322-326.

Rodrigues, M. S., \& Félix, Z. C. (2014). "ABCÊ Bulir: Uma Proposta de Software Educacional para o auxilio no Processo de Alfabetização e Desenvolvimento Cognitivo de Crianças com Síndrome de Down". In Anais do Workshop de Informática na Escola (Vol. 20, No. 1, p. 11).

Romero, T. S. (2014). "Os processos de Ensino e Aprendizagem do aluno com Síndrome de Down: um estudo no Noroeste do Paraná”, Universidade Tecnológica Federal do Paraná, Medianeira.

Schawartzman, J. S. et al. (1999). "Síndrome de down”, São Paulo: Mackenzie.

Silva, R. N. A. (2002). “A educação especial da criança com Síndrome de Down. Pedagogia em Foco". Rio de Janeiro.

Soffa, M.; Torres, P. (2009). "O processo ensino-aprendizagem mediado pelas tecnologias da informação e comunicação na formação de professores on-line". IX Congresso Nacional De Educação, EDUCERE.

Tori, R. (2009). "Desafios para o design de informação em ambientes de realidade aumentada", Infodesign: Revista Brasileira de Design da Informação, v. 6, n. 1.

Travassos, G. H. et al. (2002). "Introdução à engenharia de software experimental". UFRJ.

Veneziano, Wilson Henrique, et al.( 2013) ."Programa Participar: Software Educacional de Apoio à Alfabetização de Jovens e Adultos com Deficiência Intelectual." Brazilian Symposium on Computers in Education (SBIE). Vol. 24. No. 1, p. 477. 